\title{
Index to genera and species, Volume 3 (1992)
}

\section{Acarina, Oribatei}

See pp. 41-56: List of 465 species or subspecies from Ukraina and Czechoslovakia.

\section{Aranea}

Robertus scoticus Jackson 164, 166

Sitticus distinguendus (Simon) 164, 166

Trichonchus hackmani Millidge 164, 166

Additional 180 entries listed on pp. 165-166.

\section{Coleoptera}

Acanthocinus 200

- aedilis (Linnaeus) 195, 197-202

Acritus nigricornis (Hoffman) 95

Acrotona parens (Mulsant \& Rey) 4

Acrotrichis insularis (Mäklin) 4

Altica carduorum (Guérin-Ménéville) 1

- quercetorum saliceti Weise 1

Amara aulica (Panzer) 167, 168

- gebleri Dejean 167, 168

- helleri Gredler 167

Anthonomus brunnipennis Curtis 1, 2

- conspersus Desbrochers des Loges 7

- pedicularius (Linnaeus) 6,7

- rubi (Herbst) 1,2

Asemum striatum Linnaeus 201

Astenus pulchellus (Heer) 95

Atheta ravilla Erichson 3

Attagenus 2

- smirnovi Zhantiev 2

Bembidion monticola Sturm 7

Bledius bernhaueri Poppius 98

- fuscipes Rye 98

Brachycercus europaea Vestnik 4

- harrisella Curtis 4

Carcinops pumilio (Erichson) 95

Carphacis striatus (Olivier) 7

Carpophilus marginellus Motschulsky 95

Crioceris asparagi (Linnaeus) 2

Cryptophagus fuscicornis Sturm 3

- obsoletus Reitter 2, 3

Crypturgus spp. 208, 211

Cynaeus 95, 96

- angustus (LeConte) 96, 97

- opacus Champion 95-97

Demetrias imperialis (Germar) 3

Dendroctonus brevicornis LeConte 211

- frontalis Zimmermann 209

Deronectes 81, 82

- abyssinicus Sharp 81

- cooperi Omer-Cooper 81, 85, 86

- scotti Omer-Cooper 81, 88

Deronectes (Potamodytes) abyssinicus Sharp 83

- tellinii (Régimbart) 89

- septemvittatus (Régimbart) 90
Deronectes (Scarodytes) abyssinicus Sharp 83

Dircaea quadriguttata (Paykull) 7

Dorcatoma serra Panzer 7

Dryocoetes 209

- autographus (Linnaeus) 208, 211

Epuraea spp. 207-209, 211

- pygmaea (Gyllenhal) 208, 209

- thoracica Tournier 208

Galerucella grisescens (Joannis) 3

Gyrophaena sp. 4

- affinis Mannerheim 4

Hallomenus innatus Kangas 7

Heterocerus hispidulus Kiesenwetter 98

Hydroporus abyssinicus (Sharp) 83

- abyssinicus var. atratus Régimbart 83

- abyssinicus var. flavidus Régimbart 83

- atratus Régimbart 81

- eugeniae Zaitzew 98

- flavidus Régimbart 81

- longitarsis J. Sahlberg 98

- puberulus Lee 98

- septemvittatus Régimbart 81,90

- tellinii Régimbart 81,89

Hydroporus (Deronectes) abyssinicus (Sharp) 83

Hylobius abietis Linnaeus 195, 197-199, 201

- pales (Herbst) 201, 202

Hypocaccus rugiceps (Duftschmid) 98

Ips 209

- typographus (Linnaeus) 205-212

Laccobius sinuatus Motschulsky 7

Leptusa pulchella (Mannerheim) 208

Lionychus quadrillum (Duftschmidt) 3

Longitarsus ochroleucus (Marsham) 4

Martianus 97

- dermestoides (Chevrolat) 97

Microdota spatuloides S. Benick 98

Microrhagus lepidus Rosenhauer 7

Monochamus 195, 196, 200-202

- alternatus Hope 196, 200, 201

- carolinensis (Olivier) 201

- galloprovincialis (Olivier) 195, 197-198, 200-201

- scutellatus (Say) 201

Myrmechixenus vaporariorum Guérin-Ménéville 95

Nargus velox (Spence) 4

Nebrioporus Régimbart 81, 82, 84-88, 90-93

- abyssinicus (Sharp) 81-88,90-93

- abyssinicus var. atratus (Régimbart) 87

- capensis (Omer-Cooper) 92

- cooperi (Omer-Cooper) 82, 83-88, 90, 91

- depressus (Fabricius) 81, 93

- kilimandjarensis Régimbart 81,92

- scotti (Omer-Cooper) 83-88,90

- septemvittatus (Régimbart) 90

- solivagus (Omer-Cooper) 92

- tellinii (Régimbart) 83-91,93

- vagrans (Omer-Cooper) 92

Notiophilus aestuans Motschulsky 8

Nudobius 209

- lentus Erichsson 208 
Omalium littorale Kraaz 98

Oxyporus mannerheimi Gyllenhal 7

Perigona nigriceps (Dejean) 95

Peschetius 90

Phacophallus parumpunctatus (Gyllenhal) 95

Phloeonomus spp. 207, 208, 211

- pusillus Gravenhorst 211

- sjoebergi Strand 208

Phloeopora 211

- testacea Mannerheim 208, 211

Phytobaenus amabilis F. Sahlberg 7

Pityogenes 209, 211

- chalcographus (Linnaeus) 207, 208, 211

Placusa spp. 211

- despecta Erichson 211

- incompleta Sjöberg 208

- infima Escherich 211

- tachyporoides Waltl 211

Plegaderus spp. 208, 209

- vulneratus (Panzer) 208, 211

Potamonectes Zimmermann 81, 82

- abyssinicus (Sharp) 83, 89, 90

- cooperi (Omer-Cooper) 85

- griseostriatus DeGeer 16

- laeviventris (Reiche) 83

- scotti (Omer-Cooper) 88, 89

- septemvittatus (Régimbart) 90

- tellinii (Régimbart) 89

Quedius 209

- limbatoides Coiffat 14

- plagiatus (Gyllenhal) 207, 208, 211

Rhagium inquisitor (Linnaeus) 195, 197-199, 201-202, 208, 209, 211

Rhizophagus 209, 211

- dispar (Paykull) 208, 211

- ferrugineus (Paykull) 208, 211

- limbatus (Heer) 14

Rugilus orbiculatus (Paykull) 95

Scarodytes 82, 83, 92

- abyssinicus (Sharp) 83

Sciodrepoides alpestris Jeannel 4

Scraptia fuscula Müller 7

Sibina primita (Herbst) 3

Thamiaraea hospita (Märkel) 4

Thanasimus spp. 207-209, 211

Thanatophilus dispar (Herbst) 98

Tribolium 95

- destructor Uyttenboogart 95

Typhaea stercorea (Linnaeus) 95

Zimmermannius Guignot 92

\section{Dictyoptera}

Blattella germanica (Linnaeus) 95

\section{Diptera}

Allodia ornaticollis (Meigen) 141

Alloeostylus diaphanus (Wiedemann) 141

Bremia ciliata 140, 141

Cheilosia scutellata (Fallén) 141

Chironomus 16

Corynoptera sp. 140, 141

- blanda (Winnertz) 140

Docosia gilvipes (Walker) 141

Drosophila transversa Fallén 140-142

Exechia contaminata Winnertz 141

Fannia ciliata (Stein) 140

- difficilis (Stein) 141
Glyptotendipes 16

Limonia bifasciata (Schrank) 140

Lonchaea spp. 207-209

- bruggeri Morge 207, 208, 211

Medetera spp. 207-209, 211

- breviseta Parent 207, 208

- excellens Frey 207, 208

- signaticornis Loew 207, 208, 211

Megaselia 139, 142, 143, 147

- flava (Fallén) 140

- rubella (Schmitz) 141, 142

- rubescens Wood 141-143

- spinicincta Wood 141, 142

Meromyza Meigen 121-123

- cerealium Reuter 128

- coroonoseta Hubicka 128

- curvinervis Zetterstedt 122-124, 137

- decora Frey 123

- elbergi Fedoseeva 122-125, 137

- femorata Marquart 122

- ingrica Nartshuk sp.n. 121, 123, 125, 126, 137

- laeta Meigen 122, 123, 126, 127

- lidiae Nartshuk sp.n. 121-123, 126, 127, 137

- mosquensis Fedoseeva 122, 123, 128, 137

- nigriseta Fedoseeva 122, 123, 128, 129, 138

- nigriventris Marquart 122, 123, 128, 129, 138

- palposa Fedoseeva 122-124, 129, 130, 138

- pluriseta Péterfi 122, 123, 128-130, 138

- pratorum Meigen 122-125, 130, 131, 138

- rohdendorfi Fedoseeva 122, 123, 131, 132, 138

- rostrata Hubicka 122, 123, 131, 132, 138

- rufa Fedoseeva 122

- saltatrix Linnaeus $122,123,131,133,138$

- sibirica Fedoseeva 122, 123, 133, 134

- smirnovi Fedoseeva 122

- sororcula Fedoseeva 122, 123, 125, 131, 133, 134, 138

- triangulina Fedoseeva 122, 123, 133, 135, 138

- variegata Meigen 122-124, 135-137

- zachvatkini Fedoseeva 122

- zimzerla Nartshuk sp.n. 121, 123, 135-137, 138

Minilimosina fungicola (Haliday) 141

Muscina assimilis (Fallén) 140

Mycetophila fungorum DeGeer 140-142

- signatoides Dziedzicki 141, 142

- strobli (Lastovka) 141

Mycomya sp. 140, 142

Mydaea 142

- detrita Zetterstedt 141, 142

- discimana Malloch 140, 142

- setifemur Ringdahl 141, 142

- tincta Zetterstedt 140-142

Pegomya 139, 142, 147

- circumpolaris Ackland \& Griffiths 140, 142

- flavoscutellata (Zetterstedt) 140-142

- geniculata (Bouché) 141, 142

- incisiva Stain 140, 142

- pulchripes (Loew) 140, 142

- scapularis (Zetterstedt) 140, 142

- tabida (Meigen) 140, 142

- transversa (Fallén) 140

- vittigera (Zetterstedt) 140, 142

- zonata (Zetterstedt) 140, 142

Psychoda lobata Tonnoir 140-142

Rondaniella dimidiata (Meigen) 141

Suillia 147

- atricornis (Meigen) 141

Tephrochlamys flavipes Zetterstedt 140, 142

Trichocera saltator (Harris) 140

Ula sylvatica Meigen 140-142

Zabrachia minutissima Zetterstedt 208 
Grylloblattida (all fossil)

Aenigmidelia Sharov 22

Archidelia Sharov 22, 26

Austroidelia Riek 22

Idelia M. Zalessky 22

Idelina Storozhenko gen.n 21, 26, 38

- kamensis Storozhenko sp.n. 21, 26-28

Idelinella Storozhenko gen.n 21, 26

- macroptera Storozhenko sp.n. 21, 26, 27

Khosaridelia Storozhenko gen.n. 21,27, 28

- martynovi Storozhenko sp.n. 21, 28

Kolvidelia G. Zalessky 22

Korshakolia Sharov 22

Metidelia Martynov 21, 22, 29

Paridelia Sharov 22, 27-29

Permotermopsis Martynov 22, 36

- pectinata Martynov 38, 39

- roseni Martynov 38, 39

Permula Handlirsch 22

Rachimentomon G. Zalessky 22, 29

Sojanidelia Storozhenko gen.n. 21, 29

- fasciata Storozhenko sp.n. 21, 29-31

- fluctuosa Storozhenko sp.n. 21, 29, 34, 35

- kostinae (Sharov) 21, 29

- lineata Storozhenko sp.n. 21, 29, 36, 37

- longula Storozhenko sp.n. 21, 29, 30, 32

- maculosa Storozhenko sp.n. 21, 29, 31

- makarkini Storozhenko sp.n. 21, 29, 34, 35

- multimediana Storozhenko sp.n. 21, 29, 30, 32

- nana Storozhenko sp.n. 21, 29, 33, 34

- parvula Storozhenko sp.n. 21, 29, 33

- striata Storozhenko sp.n. 21, 29, 36, 37

- vorkutensis Storozhenko sp.n. 21, 29, 30, 32

Stenaropodites Martynov 22, 26

- magna Storozhenko sp.n. 21, 26, 27

- mistshenkoi Storozhenko sp.n. 21, 24-26

- permiakovae M. Zaleesky 22, 23

- reticulata Martynov 22, 23

- sojanensis Storozhenko sp.n. 21-24

Sylvidelia Martynov 22, 26

\section{Heteroptera}

Adelphocoris bimaculicollis Lindberg 227

Agnocoris reclairei Wagner 228

Agraptocorixa gestroi (Kirkaldy) 174

Arctocorisa sp. 174

- carinata (C. Sahlberg) 15-18

- germari (Fieber) 173

Argyrotelaenus simoni Reuter \& Poppius 223

Brachycoleus lineellus Jakovlev 227

- thoracicus Puton 227

Brachynotocoris cyprius Wagner 229

- viticinus Seidenstücker 229

Callicorixa praeusta (Fieber) 174

- producta (Reuter) 15, 16, 18

Calocoris (Calocoris) instabilis Fieber 227

- ishtar Linnavuori 227

- roseomaculatus saucius Linnavuori 227

Calocoris (Closterotomus) aqranus Linnavuori 227

Calocoris (Reuterista) tegularis Puton 227

Camponotidea fieberi Reuter 224

Capsus (Heterotoma) dalmatinus Wagner 000

Centrocorisa nigripennis (Fabricius) 174

Chorosomella jakowlewi Horváth 228

Cimex meriopterum Scopoli 000

Corisa hieroglyphica Dufour 174

- macrocephala Fieber 171
- platycnemis Fieber 171

- xanthosoma Fieber 171

Corisa (Macrocorisa) salina Thomson 171

Corisella mercenaria (Say) 174

Corixa 171, 173

- affinis Leach 171, 174

- affinis var. pallida Poisson 171

- albiceps Spinola 173

- antillarum Spinola 174

- atomaria var. conglomerata Rey 171

- carinata Sahlberg 173

- charadrina Gistel 171

- coleoptrata Fabricius 174

- dorsimaculata Spinola 174

- fossarum Zetterstedt 173

- foveiceps Spinola 174

- frigidae Linnavuori 171, 172

- geoffroyi Leach 171, 174

- graphiptera Rambur 171

- griseola Spinola 173

- hellensis Sahlberg 174

- hieroglyphica Dufour 174

- immaculata Spinola 174

- minuta Fabricius 174

- mirandella Hutchinson 171

- monticola Linnavuori 171, 172

- panzeri (Fieber) 171

- pseudaffinis Poisson 171

- punctata (Illiger) 171, 174

- quinquefasciata Spinola (= 5-fasciata) 174

- regularis Herrich-Schäffer 174

- schellembergii Spinola 174

- striata Spinola 174

- undulata Sahlberg 173

Creontiades pallidus (Rambur) 226

Cymatia coleoptrata (Fabricius) 174

Cyphodema humbaba Linnavuori 228

- rubricum Seidenstïcker 228

Cyrtopeltis (Campyloneuropsis) khalafi Seidenstücker 224

Cyrtopeltis (Nesidiocoris) tenuis Reuter 224

Deraeocoris (Camptobrochis) pallens Reuter 224

- punctulatus (Fallén) 223

- lutescens (Schilling) 224

- serenus Douglas \& Scott 223

Deraeocoris (Deraeocoris) rutilus (Herrich-Schäffer) 223

Dicyphus hyalinipennis (Burmeister) 224

Dimorphocoris 215

- eckerleini Wagner 215, 216, 220, 221

- gracilis-group 215

- lateralis-group 215

- lateralis Reuter 215, 216, 218, 220-222

- punctiger (Horváth) 215, 216, 219-222

- sari Linnavuori sp.n. 215-217, 219

- saulii Wagner 215, 217, 218

- seidenstueckeri Linnavuori 228

- servadeii Tamanini 215, 217-220

- tristis (Fieber) 215, 216, 221, 222

Eurygaster maurus (Linnaeus) 111, 112

- testudinarius Geoffroy 111, 112

Eurystylus bellevoyei (Reuter) 227

Gerris najas DeGeer 15

Globiceps syriacus Wagner 230

Grypocoris fieberi Douglas \& Scott 227

Halticus beganus Linnavuori 229

- darbandikhanus Linnavuori 229

- macrocephalus Fieber 229

Hesperocorixa linnaei (Fieber) 174

- sahlbergi (Fieber) 174

Heterotoma meriopterum (Scopoli) 230

Holcostethus vernalis (Wolff) 
Liocoris tripustulatus (Fabricius) 228

Lygus pratensis pratensis (Linnaeus) 228

- pratensis osmanus Wagner 228

Macrolophus caliginosus Wagner 224

- nubilus (Herrich-Schäffer) 224

Megacoelum brevirostre Reuter 226

- irbilanum Linnavuori 227

- quercicola Linnavuori, 1965227

- quercicola Linnavuori, 1984227

- tricolor Wagner 227

Micronecta griseola Horváth 173, 174

- leucocephala (Spinola) 174-175

- scholtzi (Fieber) 174

- scutellaris pubescens Hutchinson 173

Nemocoris falleni R. F. Sahlberg 7, 8

Neosapinnius phytocoroides Wagner 226

Notonecta striata Linnaeus 174

Orthocephalus tenuicornis (Mulsant \& Rey) 228

Orthotylus (Melanotrichus) arabicus Wagner 230

- argenteopunctatus Wagner 229

- flavosparsus (C. Sahlberg) 229

- haloxyloni Wagner 229

- haloxyloni abbreviatus Linnavuori 230

- hirtulus Wagner 229

- jordanicus Wagner 230

- nigricollis Wagner 230

- omanensis Linnavuori 229

Orthotylus (Orthotylus) cornupunctus Ghauri 229

- macrophthalmus Wagner 229

- priesneri Schmidt 229

- prunicola Linnavuori 229

Parasigara multilineata de Sallier Dupin 171

Phytocoris (Eckerleinius) anabasidis Linnavuori 226

- artemisiae Linnavuori 226

- enmerkar Linnavuori 225

Phytocoris (Eriamiris) djerablisus Linnavuori 226

- echinopis Linnavuori 226

- gilgamesh Linnavuori 226

- lugalbanda Linnavuori 226

- thymi Linnavuori 226

Phytocoris (Ktenocoris) poecilus Wagner 225

- tridens Wagner 225

- ulmi (Linnaeus) 225

Phytocoris (Stictophytocoris) meridionalis Herrich-Schäffer 225

- tristis Linnavuori 225

Piesma salsolae (Becker) 5

Piezocranum corvinum Puton 228

Pithanus marshalli Douglas \& Scott 224

Plagiotylus dispar Reuter 228

Pseudoloxops guttulatus Kerzhner \& Muminov 230

Sigara 173

- argentiniensis chichinal Bachmann 174

- atomaria Illiger 171

- distincta (Fieber) 174

- forciceps (Spinola) 174

- hellensii (C. Sahlberg) 174

- indica (Hutchinson) 174

- lateralis (Leach) 174

- leucocephala Spinola 174

- limitata limitata (Fieber) 174

- minuta Fabricius 174

- minutissima Linnaeus 174

- nigrolineata nigrolineata (Fieber) 173

- rehi Jaczewski 174

- semistriata (Fieber) 173, 174

- striata Fabricius sensu Schellenberg 174

- striata (Linnaeus) 173

- undulata Fallén 173

- wahlbergi Lundblad 173
Sigara (Vermicorixa) lateralis (Leach) 174

Stenodema 117

- calcaratum (Fallén) 225

- turanicum Reuter 225

- virens (Linnaeus) 117, 225

Stethoconus cyrtopeltis Flor 224

Strongylocoris enki Linnavuori 229

Taylorilygus pallidulus (Blanchard) 228

Trigonotylus brevipes Jakovlev 225

- pallidicornis Reuter 225

- pulchellus (Hahn) 225

Zanchius alatanus Hoberlandt 230

- breviceps (Wagner) 230

\section{Homoptera}

Acyrthosiphon caraganae (Cholodkovsky) 62

- hertae Hille Ris Lambers 62, 63

- knechteli (Börner) 62

- malvae (Mosley) 62

- pisum (Harris) 62

Anoecia corni (Fabricius) 59

Anuraphis farfarae (Koch) 64, 77

Aphis brohmeri Börner 64

- callunae Theobald 64

- comari Prior \& Stroyan 64

- coronillae Ferrari 64

- craccae Linnaeus 64

- epilobii Kaltenbach 64

- euphorbiae Kaltenbach 64, 77

- fabae Scopoli s. lat. 64

- fabae s. str. 64

- fabae cirsiiacanthoidis Scopoli 64

- farinosa Gmelin 64

- frangulae Kaltenbach 65

- frangulae beccabungae Koch 65

- hieracii Schrank 65

- idaei van der Groot 65

- mammulata Gimingham \& Hille Ris Lambers 65, 77

- mirifica (Börner) 65

- nasturtii Kaltenbach 65

- pomi DeGeer 65

- pseudocomosa 65

- salicariae Koch 65

- sanguisorbae Schrank 65,77

- sarothamni Franssen 66,77

- schneideri (Börner) 66

- sedi Kaltenbach 66

- subnitida (Börner) 66

- taraxacicola (Börner) 66

- ulmariae Schrank 66

- urticata Gmelin 66

Aphis (Toxopterina) vandergooti (Börner) 66

Aspidaphium cuspidati Stroyan 66, 67

- escherichi (Börner) 66, 67

Atheroides serrulatus Haliday 61

Aulacorthum solani (Kaltenbach) 66

Baizongia pistaciae (Linnaeus) 5

Betulaphis spp. 60

- brevipilosa Börner 60

- quadrituberculata (Kaltenbach) 60

Brachycaudus (Acaudus) cardui (Linnaeus) 66

- populi (Del Guercio) 67

Brachycaudus (Brachycaudus) helicrysi (Kaltenbach) 67

Brachycaudus (Thuleaphis) rumexicolens (Patch) 67

Brevicoryne brassicae (Linnaeus) 67, 71

Calaphis betulicola (Kaltenbach) 60

- flava Mordvilko 60

Callipterinella calliptera (Hartig) 60

- tuberculata (von Heyden) 60 
Capitophorus carduinus (Walker) 67, 77

- hippophaes (Walker) 67

- horni gynoxantha Hille Ris Lambers 67

- similis van der Groot 67

Cavariella aegopodii (Scopoli) 67-69

- archangelicae (Scopoli) 67

- cicutae (Koch) 69

- intermedia Hille Ris Lambers 68, 69

- konoi Takahashi 69

- pastinacae (Linnaeus) 69

- rutila Mamontova 69

- theobaldi (Gillette \& Bragg) 69

Ceruaphis eriophori (Walker) 69

Chaetosiphon (Pentatrichopus) potentillae (Walker) 69

Chaitophorus nigricantis Pintera 61,77

- populeti (Panzer) 61

- tremulae Koch 61

Cinara costata (Zetterstedt) 76

- diversiseta Börner 76

- juniperi (DeGeer) 76

- nuda (Mordvilko) 76

- piceae (Panzer) 76

- pilicornis (Hartig) 77

- pilosa (Zetterstedt) 77

- pinea (Mordvilko) 77

- pini (Linnaeus) 77

- pinihabitans (Mordvilko) 77

- pruinosa (Hartig) 77

- viridescens (Cholodkovsky) 77

Corylobium avellanae (Schrank) 69, 77

Cryptomyzus galeopsidis (Kaltenbach) 69

- ribis (Linnaeus) 69

Decorosiphon corynothrix Börner 69

Diuraphis (Diuraphis) muehlei (Börner) 69

Diuraphis (Holcaphis) calamagrostis (Ossiannilsson) 69, 77

- frequens (Walker) 69

Dysaphis (Dysaphis) anthrisci Börner 69

- ranunculi (Kaltenbach) 69

Dysaphis (Pomaphis) plantaginea (Passerini) 69, 77

- sorbi (Kaltenbach) 70

Elatobium abietinum (Walker) 115

- plagiator (Nees) 115, 116

Eriosoma (Schizoneura) ulmi (Linnaeus) 59

Eucallipterus tiliae (Linnaeus) 60

Euceraphis betulae (Koch) 60

- punctipennis (Zetterstedt) 60

Eulachnus agilis (Kaltenbach) 77

Forda formicaria (von Heyden) 60

- marginata Koch 60

Galiobium langei (Börner) 70, 77

Geoica setulosa (Passerini) 5

Glyphina betulae (Linnaeus) 59

Gootiella tremulae Tullgren 59

Hayhurstia atriplicis (Linnaeus) 71

Hyadaphis foeniculi (Passerini) 70

- passerini (del Guercio) 70

- tataricae (Aizenberg) 71, 77

Hyalopteroides humilis (Walker) 71

Hyalopterus pruni (Geoffroy) 71

Hydaphias mosana Hille Ris Lambers 71

Hyperomyzus (Hyperomyzus) lactucae (Linnaeus) 71

- pallidus Hille Ris Lambers 71

Hyperomyzus (Hyperomyzella) rhinanthi (Schouteden) 71

Hyperomyzus (Neonasonovia) zirnitsi Hille Ris Lambers 72

Iziphya leegei Börner 60

Lachnus roboris (Linnaeus) 76, 77

Liosomaphis berberidis (Kaltenbach) 72

Longicaudus trirhodus (Walker) 72

Macrosiphoniella artemisiae (Boyer de Fonscolombe) 72

- millefolii (DeGeer) 72
- sejuncta (Walker) 72

- tanacetaria (Kaltenbach) 72

- tapuscae (Hottes \& Frison) 72

- usquertensis Hille Ris Lambers 72

Macrosiphum 62

- cholodkovskyi (Mordvilko) 72

- gei (Koch) 72

- rosae (Linnaeus) 72, 115

Maculolachnus submacula (Walker) 76

Megoura viciae Buckton 72

Melanaphis luzulella (Hille Ris Lambers) 72, 73

Metopeurum fuscoviride Stroyan 72

Metopolophium 62

- dirhodum (Walker) 72

- festucae (Theobald) 73

- tenerum Hille Ris Lambers 73

Microlophium carnosum (Buckton) 73

Mindanus abietinus Koch 59

Myzaphis rosarum (Kaltenbach) 73

Myzocallis coryli (Goeze) 60

- myricae (Kaltenbach) 61

Myzosiphon staphyleae (Koch) 73

Myzus borealis Ossiannilsson 73

- cerasi (Fabricius) 73

Nasonovia pilosellae (Börner) 73

- ribisnigri (Mosley) 73

Neoamphorophora ledi (Wahlgren) 73

Ovatus crataegarius (Walker) 73

Pachypappa spp. 59

- populi (Linnaeus) 59

- tremulae (Linnaeus) 59

Pachypappella lactea (Tullgren) 59

Paracletus cimiciformis (von Heyden) 60

Pemphigus bursarius (Linnaeus) 60

- populinigrae (Schrank) 60

Periphyllus aceris (Linnaeus) 61

- coracinus (Koch) 61

- lyropictus (Kessler) 62

- testudinaceus (Fernie) 62

Phorodon humuli (Schrank) 73

Phyllaphis fagi (Linnaeus) 61, 77

Pineus pini (Gmelin) 59

Prociphilus (Stagona) pini (Burmeister 60

- xylostei (DeGeer) 60

Protrama flavescens (Koch) 5,6

- ranunculi (Del Guercio) 77

Pseudorhopalosiphoninus calthae (Koch) 74

Pterocallis alni (DeGeer) 61

- maculatus (von Heyden) 61

Pterocomma jacksoni Theobald 62

- pilosum Buckton 62

- pilosum konoi Hori 62

- rufipes (Hartig) 62

- salicis (Linnaeus) 62

Rhopalomyzus lonicerae (von Siebold) 74

Rhopalosiphoninus ribesinus (van der Groot) 74

Rhopalosiphum insertum (Walker) 74

- maidis (Fitch) 74

- padi (Linnaeus) 74

Sacchiphantes abietis (Linnaeus) 59

Schizaphis aggrostis Hiulle Ris Lambers 74

- borealis Tambs-Lyche 74,77

Schizolachnus pineti (Fabricius) 77

Sitobion (Metobion) graminearum (Mordvilko) 75

Sitobion (Sitobion) avenae (Fabricius) 75

- equiseti Holman 75

- fragariae (Walker) 75

Smynthurodes betae Westwood 6

Subacyrthosiphon cryptobium Hille Ris Lambers 76

Subsaltusaphis aquatilis (Ossiannilsson) 61, 77

- rossneri (Börner) 61

Symydobius oblongus (von Heyden) 61

Tetraneura ulmi (Linnaeus) 59

Thecabius affinis (Kaltenbach) 60

Thelaxes dryophila (Schrank) 59 
Therioaphis luteola (Börner) 61

- trifolii (Monell) 61

Thripsaphis caricicola (Mordvilko) 61

Thripsaphis (Trichocallis) caricis (Mordvilko) 61

- cyperi (Walker) 61

- verrucosa Gillette 61

Trama troglodytes von Heyden 77

Trichosiphonaphis corticis (Aizenberg) 76, 78

Tubaphis ranunculina (Walker) 76

Tuberculatus (Tuberculoides) annulatus (Hartig) 61

Uroleucon 62

Uroleucon (Uroleucon) achilleae (Koch) 76

- cichorii grossum (Hille Ris Lambers) 76

- cirsii (Linnaeus) 76

Uroleucon (Uromelan) jaceae (Linnaeus) 76

- solidaginis (Fabricius) 76

- taraxaci (Kaltenbach) 76

Wahlgreniella vaccinii (Theobald) 76

\section{Hymenoptera}

Agrypon canaliculatum (Ratzeburg) 159

- rugifer (Thomson) 159

Alysia Latreille 142, 143

- frigida Haliday 140, 142, 147

- luciella Stelfox 140

- sophia Haliday 142

- tipulae Scopoli 142

Anisocyrta perdita Haliday 140

Aspilota Foerster 139, 140, 142-144, 146

- aequabilis Tobias sp.n. 139

- breviantenata Tobias 142

- falsifica Stelfox \& Graham 142

- fungicola Tobias sp.n. 139

- fuscicornis Haliday 142

- jakovlovi Tobias sp.n. 139

- karelica Tobias sp.n. 139

- kempei Hedqvist 143

- mandibularis Tobias 142

- spiritalis Tobias sp.n. 139

- stigmatica Tobias sp.n. 139

Aspilota (Aspilota) jakovlevi Tobias sp.n. 141, 143-145

- karelica Tobias sp.n. 141, 143, 144

- laevinotum Tobias 143

Aspilota (Dinotrema) aequalis Tobias sp.n. 141, 143, 146

- erythropa Foerster 145

- fungicola Tobias sp.n. 141, 143, 145, 146

- kempei Hedqvist 140, 146

- oleracea Tobias 145

- pratensis Van Achtenberg 146

- sessilis Van Achterberg 141

- stigmatica Tobias sp.n. 141, 143, 145

- taurica Telenga 141

- varimembris Fischer 141

Aspilota (Synaldis) megastigma Fischer 144

- spiritalis Tobias sp.n. 141, 143, 144

Blacus (Blacus) exilis Nees 140-142

Bracon (Glabrobracon) pineti Thomson 140, 141

Cidaphus alarius (Gravenhorst) 159

- brischkei (Szépligeti) 159

Cosmophorus regius Nietzabitowski 208

Diadegma armillata (Gravenhorst) 160

Dinotiscus eupterus Walker 208

Dinotrema Foerster 140

Diprion simile (Hartig) 153

Doryctes mutillator Thunberg 140, 141

Ephedrus (Ephedrus) cerasicola Stary 113

- koponeni Halme sp.n. 113

- plagiator (Nees) 113

- vaccinii Gärdenfors 113

Formica 62

Gilpinia hercyniae (Hartig) 149

Idiasta sp. 140

Karpinskiella pityophtori Boucek 208, 211

Lasius flavus (Fabricius) 5, 6

- niger (Linnaeus) 5

Limneria neglecta Holmgren 159
Mesochorus alarius Gravenhorst 159

- albipes Thomson 159, 160

- anomalus Holmgren 160

- crassimanus Holmgren 160

- dimidiatus Holmgren 160

- fulvus Thomson 160

- globulator (Thunberg) 159-161

- lapponicus Thomson 159-161

- novus Kiss 159, 160

- orbitalis Holmgren 160, 161

- pictilis Holmgren 162

- suecicus Dalla Torre 160

- temporalis Thomson 159-161

- velox Thomson 159, 162

Monomorium pharaonis (Linnaeus) 6

Neodiprion sertifer (Geoffroy) 149-153

Ophthalmochorus brischkei (Szépligeti) 159

Peristenus stenodemae Loan 117-119

Phaenocarpa Foerster 142, 143

- pratellae Curtis 140, 142

Phobocampe bicingulata (Gravenhorst) 159

- neglecta (Holmgren) 159

Rhopalicus tutele Walker 208, 211, 212

Rhopalophorus clavicornis Wesmael 208

Roptroderus brevicornis Thomson 208, 211

- xylophagorum Ratzeburg 207, 208, 211

Synaldis Foerster 140

Tapinoma melanocephalum (Fabricius) 6

Tomicobia seitneri Ruschka 208, 211, 212

Ussurinus nobilis Saarinen 7

\section{Lepidoptera}

Achroia grisella (Fabricius) 103

Adela leucocerella (Scopoli) 100

Agonopterix bipunctosa (Curtis) 99, 101

- parilella (Treitschke) 101

Agrius convolvuli (Linnaeus) 7

Alcis maculatus (Staudinger) 103

Amphipyra livida (Denis \& Schiffermüller) 6

Archimeesia irinae Savenkov 100

Aristotelia caeruleopictella baltica Sulcs 102

Autographa buraetica (Staudinger) 7

Bembecia triannuliformis (Freyer) 103

Biselachista 180, 181

- freyi Staudinger 180, 181

- igaloensis Amsel 181

- serricornis Stainton 180, 181

- spinigera Sruoga 180

- zonulae Sruoga 177, 180, 181

Bucculatrix 190

- noltei Petry 99, 100

Capperia trichodactyla (Denis \& Schiffermüller) 103

Cauchas rufimitrella (Scopoli) 100

Celypha flavipalpana (Herrich-Schäffer) 11, 13

- rurestrana (Duponchel) 13

Clepsis spectrana (Treitschke) 12

Cnephasia alticolana (Herrich-Schäffer) 102

Coleophora alnifoliae Barasch 12

- badiipennella (Duponchel) 11, 12

- caelebipennella Zeller 12

- carelica Hackman 101

- conspicuella Zeller 12

- conyzae Zeller 99, 101

- gardesanella Toll 99, 101

- gryhipennella (Hübner) 101

- limosipennella (Duponchel) 12

- milvipennis Zeller 12

- ptarmicia Walsingham 101

- scabrida Toll 101

- vibicigerella Zeller 11, 12

Cosmiotes consortella (Stainton) 194

- pallens Sruoga 194

- vakshi Sruoga 194

Depressaria chaerophylli Zeller 11, 12

- olerella Zeller 12

Dichelia Guenee 12 
Dichrorampha cinerascens (Danilevsky) 102

- uralensis (Danilevsky) 99, 102

Ectoedemia albibimaculella (Larsen) 99, 100

- klimeschi (Skala) 100

- longicaudella Klimesch 99, 100

- septembrella (Stainton) 101

Elachista 105, 158, 191-193

- abiscoella Bengtsson 180

- albicapilla Höfner 186

- albifrontella (Hübner) 184

- alpinella Stainton 183

- anserinella Zeller 187

- baikalica Kaila sp.n. 177, 182-184

- bedellella (Sircom) 192, 193

- bedellella-group 190

- biatomella (Stainton) 180

- bicingulella Sruoga 184

- bifasciella -group 155, 158

- bimaculata Parenti 177, 188, 189

- bisulcella (Duponchel) 193

- cerusella (Hübner) 188

- coeneni Traugott-Olsen 193

- constitellla Frey 189

- diederichsiella Hering 182

- dispilella Zeller 191, 192

- dispilella-complex 191

- eskoi Kyrki \& Karvonen 155, 156

- esmeralda Kaila sp.n. 177, 190

- filicornella Kaila sp.n. 177, 191, 192

- flavescens Parenti 191

- gleichenella (Fabricius) 179

- grandiferella Sruoga 185

- griseella (Duponchel) 182

- heringi Rebel 189

- ilicrina Falkovitsh 189

- ingvarella Traugott-Olsen 184

- irenae Buszko 185, 186

- kilmunella Stainton 155-158

- leifi Kaila \& Kerppola sp.n. 155-158

- luticomella Zeller 182, 183

- manca Falkovitsh 189

- monosemiella Rössler 187, 188

- multipunctata Sruoga 189

- nolckeni Sulcs sp.n. 105-107, 190

- parasella Traugott-Olsen 155, 156, 158, 182

- phragmitella Sruoga 193

- pullicomella Zeller 193

- revinctella Zeller 193

- rudectella Stainton 192

- spumella Caradja 191

- squamosella (Herrich-Schäffer) 190

- subalbidella Schläger 193

- subnigrella Douglas 184

- subocellea (Stephens) 105, 106

- talgarella Kaila sp.n. 177, 185-187

- triatomea (Haworth) 188

- zernyi Hartig 101

Elachistoides sinevi Sruoga 178, 190

Elaphria venustula (Hübner) 7

Epinotia indecorana (Zetterstedt) 99, 102

Epirrita autumnata (Borkhausen) 159

Eupithecia cauchiata (Duponchel) 103

- ochridata Pinker 99, 103

Eupoecilia sanguisorbana (Herrich-Schäffer) 102

Heliophobus kitti Schawerda 104

Hydraecia petasitis Doubleday 104

Kumia integra Falkovitsh 178

Laspeyresia 141

- strobilella (Linnaeus) 141

Leucoptera lustratella (Herrich-Schäffer) 101

Maculinea teleidus (Bersträsser) 103

Metzneria aestivella (Zeller) 99, 102

Monochroa divisella (Douglas) 102

Noctua janthe (Borkhausen) 9*, 10

- janthina 9*, 10

Oligia fasciuncula (Haworth) 104

Opostega salaciella (Treitschke) 11

Ornixola caudulatella (Zeller) 100
Pammene clanculana (Tengström) 99, 102

Parasyndemis Obratzov 12-13

Pelosia obtusa (Herrich-Schäffer) 103

Perittia biloba Sruoga 178

- petrosa Sruoga 178

Phyllonorycter 190

- anderidae (W. Fletscher) 100

Pristerognatha fuligana (Denis \& Schiffermüller) 13

Pyrgus serratulae (Rambur) 103

Schrankia taenialis (Hübner) 103

Scrobipalpa klimeschi Povolny 102

- nitentella (Fuchs) 99, 102

- proclivella (Fuchs) 102

Scythris cicadella (Zeller) 101

- palustris (Zeller) 102

Stephensia brunnichella (Linnaeus) 179

- jalmarella Kaila sp.n. 177, 179

- staudingeri Nielsen \& Traugott-Olsen 179

Stigmella betulicola (Stainton) 99

- myrtillella (Stainton) 99

- nanivora (W. Petersen) 99

- oxycanthella (Stainton) 99

- poterii (Stainton) 99

- pretiosa (Heinemann) 99

Swammerdamia caesiella (Hübner) 11

- passerella (Zetterstedt) 11,12

- pyrella (de Villers) 11, 12

Syndemis Hübner 13

- histrionana (Frölich) 11-13

Teleiodes notatella (Hübner) 12

- paripunctella (Thunberg) 12

- wagae (Nowicki) 11, 12

Tinea cerusella Hübner 188

- cerussella Denis \& Schiffenmüller 188

- pallescentella Stainton 99, 100

- steueri G. Petersen 100

Tischeria heinemanni Wocke 100

Trifurcula headlayella (Stainton) 100

- subnitidella (Duponchel) 11

Xylomoia strix Mikkola 104

Yponomeuta evonymellus Linnaeus 160

\section{Nematoda}

Bursaphelenchus 196

- mucronatus Mamiya \& Enda 195-202

- xylophilus (Steiner \& Buhrer) 195-196, 200-202

Neoaplectana carpocapsae Weiser 201

\section{Pseudoscorpionida}

Chernes 209

- cimicoides (Fabricius) 208, 211
* Note the following correction: In Fig. 1, p. $9, N$. janthe is
on the right, $N$. janthina on the left. 